\begin{abstract}
A dysregulation of the redox homoeostasis has been reported in various neoplastic disorders. Malondialdehyde/4-hydroxy-2,3-nonenal (MDA/HNE) and protein carbonyl groups represent in vivo indexes of lipid peroxidation and protein oxidation, respectively, suitable to investigate radical-mediated physio-pathological conditions.

We evaluated MDA/HNE and protein carbonyl groups in sera of untreated Hodgkin's lymphoma (HL) patients in advanced disease stages, in order to quantify the oxidative stress.

HL patients displayed significantly higher levels of both MDA/HNE and protein carbonyl groups as compared with healthy controls.

This is the first evidence that a strong increase in $\mathrm{HL}$ is one of the most common haematological malignancies, representing approximately $30 \%$ of all lymphomas in the circulating protein carbonyl content in HL. These findings may contribute to a better definition of the redox homoeostasis dysregulation in HL.
\end{abstract}

Key words: Hodgkin's lymphoma, Malondialdehyde/4hydroxy-2,3-nonenal, Protein carbonyl groups, Lipid peroxidation, Protein oxidation

\section{Lipid peroxidation and protein oxidation in patients affected by Hodgkin's lymphoma}

\author{
F. Morabito ${ }^{1}$, M. Cristani ${ }^{2}$, A. Saija ${ }^{2}$, C. Stelitano ${ }^{1}$, \\ V. Callea ${ }^{1}$, A. Tomaino ${ }^{2}$, P. L. Minciullo ${ }^{3}$ and \\ S. Gangemi ${ }^{3, C A}$
}

${ }^{1}$ Bone Marrow Transplant Unit, Azienda Ospedaliera Bianchi-Melacrino-Morelli, Reggio Calabria, Italy; ${ }^{2}$ Department Farmaco-Biologico and ${ }^{3}$ Division and School of Allergy and Clinical Immunology, Department of Human Pathology, University of Messina, via Centonze 200, is. 98, 98123 Messina, Italy

\footnotetext{
${ }^{\mathrm{CA}}$ Corresponding Author

Tel: +3990716070

Fax: +39906782336

E-mail: sgangemi@eniware.it
}

Hodgkin's lymphoma (HL) represents approximately $30 \%$ of all malignant lymphomas; despite the recent treatment success rate, the pathogenesis of HL is still largely unknown.

Lipid peroxidation is a well-established mechanism of cellular injury in both plants and animals. This process, leading to the production of lipid peroxides and their by-products, and ultimately the loss of membrane function and integrity, is widely accepted to be involved in the pathogenesis of several human diseases.

Malondialdehyde (MDA) and 4-hydroxy-2,3-nonenal (HNE) are end-products derived from peroxidation of polyunsaturated fatty acids and related esters. In contrast to free radicals, aldehydes are relatively stable and therefore able to diffuse within or out of the cell and to attack targets distant from the site of original free-radical-initiated events. These aldehydic molecules are considered ultimate mediators of toxic effects elicited by oxidative stress occurring in biological materials. ${ }^{1}$

Measurement of MDA levels in plasma or serum provides a suitable in vivo index of lipid peroxidation and represents a non-invasive biomarker of oxidative stress often clinically employed to investigate radical-mediated physiological and pathological conditions. ${ }^{1}$

The action of reactive oxygen species on proteins has been widely demonstrated to increase the formation of carbonyl groups. ${ }^{2}$ Moreover, carbonyl stress may be due to the damaging effect of various monodicarbonyls (such as MDA and HNE) and of hypochlorous acid (whose production is catalysed by myeloperoxidase in neutrophils) on proteins. However, in contrast to lipid peroxidation, protein oxidation does not have the features of chain reactions. Also the level of carbonyl groups in circulating proteins is considered a useful marker of oxidative stress, which may have some advantages in comparison with the measurement of other parameters; in fact, these oxidation products are formed relatively early and are more stable.

In this study, we analysed the serum levels of MDA/HNE and of protein carbonyl groups as parameters of lipid peroxidation and protein oxidation, respectively, in order to quantify the oxidative stress in untreated HL patients in advanced disease stages.

Fifteen previously untreated HL patients in III-IV disease stages (four males and 11 females) with a median age of 29 years (range, 19-65 years) entered this study. Sera from 20 sex-matched and agematched normal subjects were also included in this study as controls.

Sera were obtained from peripheral blood allowed to clot at room temperature for $2 \mathrm{~h}$, separated by centrifugation at $200 \times \mathrm{g}$ for $15 \mathrm{~min}$ in a $4235 \mathrm{~A}$ centrifuge (ALC Int. S.r.L., Milan, Italy), and stored at $-80^{\circ} \mathrm{C}$ until used. 
Serum MDA/HNE levels were measured by means of a colorimetric assay specific for MDA and carried out using a commercially available kit (CalbiochemNovabiochem Corporation, La Jolla, CA, USA). This method employs a chromogenic reagent, which reacts with $\mathrm{MDA} / \mathrm{HNE}$ at $45^{\circ} \mathrm{C}$, yielding a stable chromophore with maximal absorbance at $586 \mathrm{~nm}$.

The serum content of protein carbonyl groups was evaluated with use of the Levine method; briefly, $100 \mu \mathrm{l}$ of serum was incubated with $100 \mu \mathrm{l}$ of a $20 \mathrm{mM}$ 2,4-dinitro-phenylhydrazine solution for $60 \mathrm{~min}$. Then the proteins were precipitated from the solution with the use of $20 \%$ trichloroacetate; the protein pellet was washed three times with ethanol and ethyl acetate and resuspended in $1 \mathrm{ml}$ of $6 \mathrm{M}$ guanidine at $37^{\circ} \mathrm{C}$ for $15 \mathrm{~min}$. The carbonyl content was determined from the absorbance at $366 \mathrm{~nm}$ (molar absorption coefficient, 22,000 $\mathrm{M}^{-1} / \mathrm{cm}$ ).

All statistical calculations were performed using the statistical package SPSS for Windows (release 11.5, 2002 software; SPSS UK, Woking, UK). The nonparametric Mann-Whitney U-test was employed to test the differences between two groups. Data were expressed as the mean \pm standard deviation. $p<0.05$ was considered significant.

MDA/HNE serum levels were significantly higher in HL patients as compared with controls (Fig. 1A) $(64.62 \pm 26.81 \mathrm{nmol} / \mathrm{ml}$ versus $14.88 \pm 1.81 \mathrm{nmol} / \mathrm{ml}$, $p<0.0001)$. Similarly, the serum content of protein carbonyl groups was significantly more elevated in HL patients than in normal subjects $(44.21 \pm 18.71$ $\mathrm{nmol} / \mathrm{ml}$ versus $25.37 \pm 7.35 \mathrm{nmol} / \mathrm{ml}, \quad p<0.001)$ (Fig. 1B).

It is well known that oxidative stress may be associated not only with initiation, but also with promotion and progression in the multistage carcinogenesis model.

In fact, the abnormal production of cellular oxidants or the imbalance of the antioxidant control systems have been linked to mutation (induced by oxidant-induced DNA damage), as well as modification of gene expression. Moreover, several signal transduction pathways, such as activator protein-1 and nuclear factor- $\kappa \mathrm{B}$, are known to be activated by reactive oxygen species, and they lead to the transcription of genes involved in cell growth regulatory pathways. ${ }^{3}$

With regard to MDA/HNE serum levels, our results are in agreement with those of Guven et al., showing significantly higher MDA concentrations in sera of HL patients compared with healthy donors. ${ }^{4}$ Moreover, a high MDA concentration has been shown to be associated with a dysregulation of antioxidant system (i.e. low levels of selenium, zinc and glutathione peroxidase activity in HL). ${ }^{4}$

High levels of protein carbonyl groups have been observed in several diseases, such as Alzheimer's
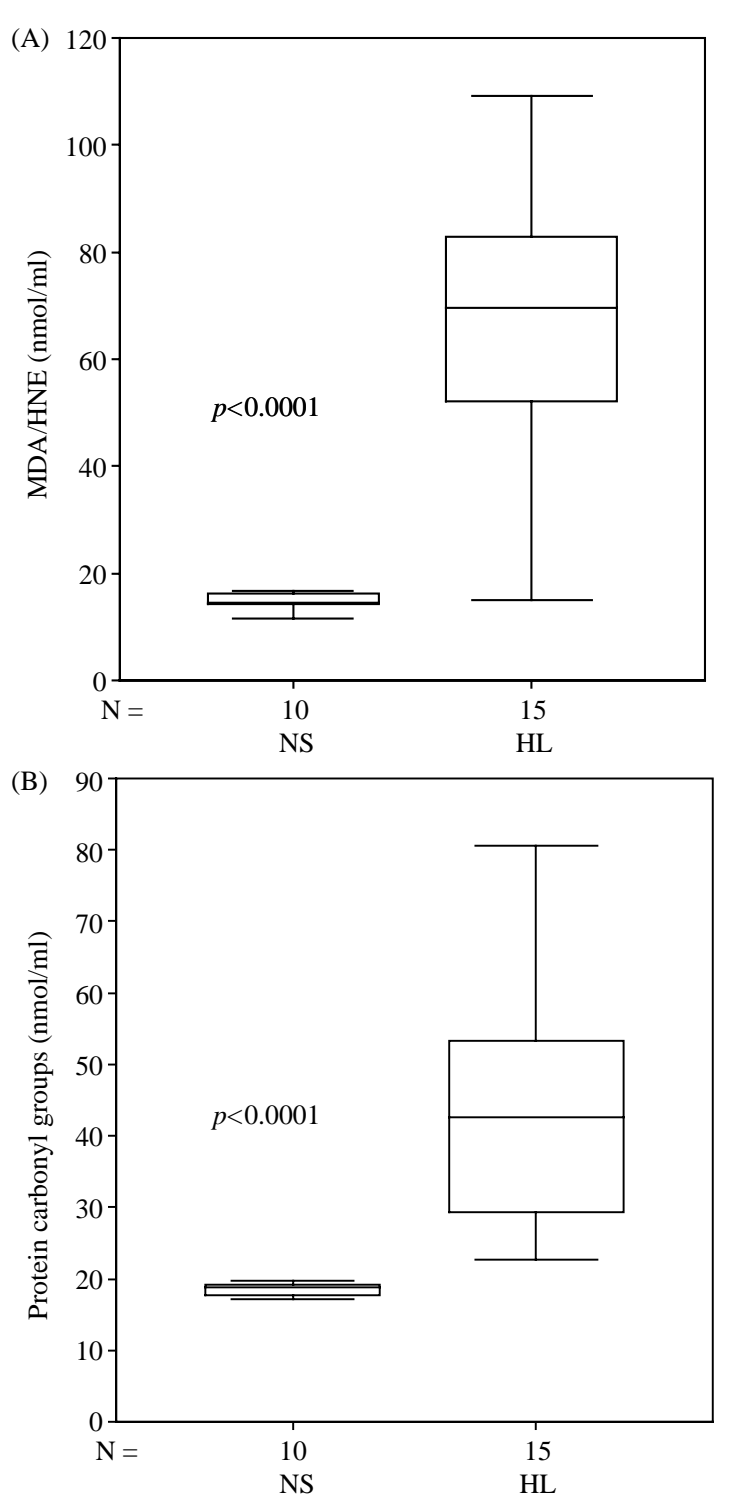

FIG. 1. Serum levels of (A) MDA/HNE and (B) protein carbonyl groups detected in normal subjects (NS) and patients affected by HL.

disease, rheumatoid arthritis, diabetes, sepsis, chronic renal failure, and in some malignancies.

To our knowledge, data on blood protein carbonyl groups in HL were not currently available. Here we show significantly higher circulating protein carbonyl groups in HL patients, suggesting that HL patients suffer from oxidative stress strictly affecting proteins.

Although it is rather out of the question to unravel the issue of whether oxidative stress, in particular protein oxidation, represents one of the causes or a consequence of HL development, several models of experimental tumourigenesis have shown that elevated levels of protein carbonyl groups are early targets of carcinogenesis.

In conclusion, we have herein confirmed the involvement of lipid peroxidation and provided the first evidence of a strong increase in circulating protein carbonyl groups in HL patients. Consistent 
with our findings, Goswami and Koner ${ }^{5}$ measured a higher level of protein carbonylation in platelets from HL than in those from controls. Our data may contribute to a better definition of the redox homoeostasis dysregulation in HL and suggest that antioxidants may be considered a support therapy for this disease.

\section{References}

1. Meagher EA, FitzGerald GA. Indeces of lipid peroxidation in vivo: strenghts and limitation. Free Radic Biol Med 2000; 28: 1745-1750.
2. Dalle-Donne I, Rossi R, Giustarini D, Milzani A, Colombo R. Protein carbonyl groups as biomarkers of oxidative stress. Clin Chim Acta 2003; 329: $23-38$.

3. Klaunig JE, Kamendulis LM. The role of oxidative stress in carcinogenesis. Annu Rev Pharmacol Toxicol 2004; 44: 239-267.

4. Guven M, Ozturk B, Sayal A, Ozet A. Lipid peroxidation and antioxidant system in the blood of patients with Hodgkin's disease. Clin Biochem 2000; 33: 209-212.

5. Goswami K, Koner BC. Level of sialic acid residues in platelet proteins in diabetes, aging and Hodgkin's lymphoma: a potential role of free radicals in desialylation. Biochem Biophys Res Commun 2002; 297: 502-505.
Received 11 August 2004

Accepted 17 August 2004 


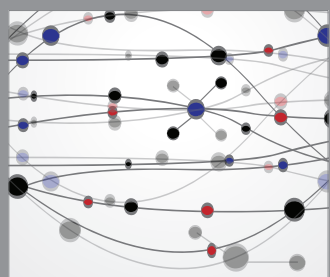

The Scientific World Journal
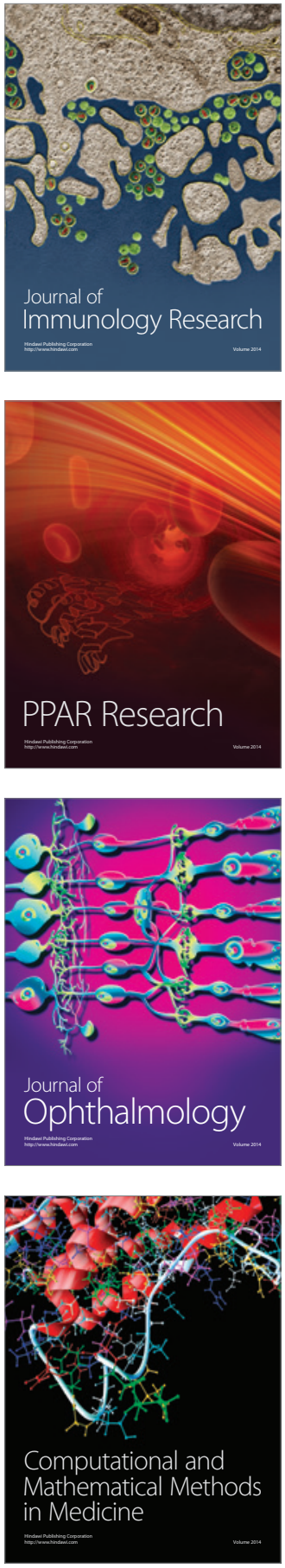

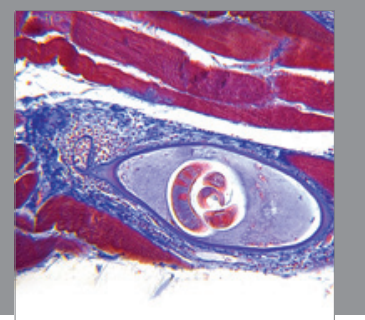

Gastroenterology

Research and Practice
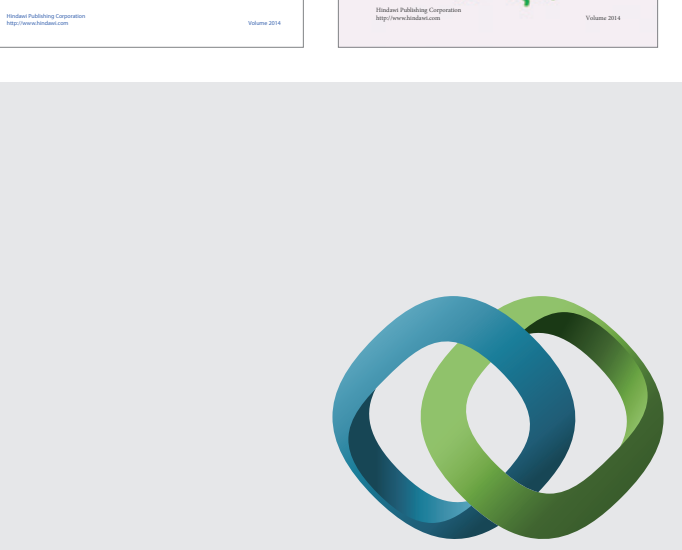

\section{Hindawi}

Submit your manuscripts at

http://www.hindawi.com
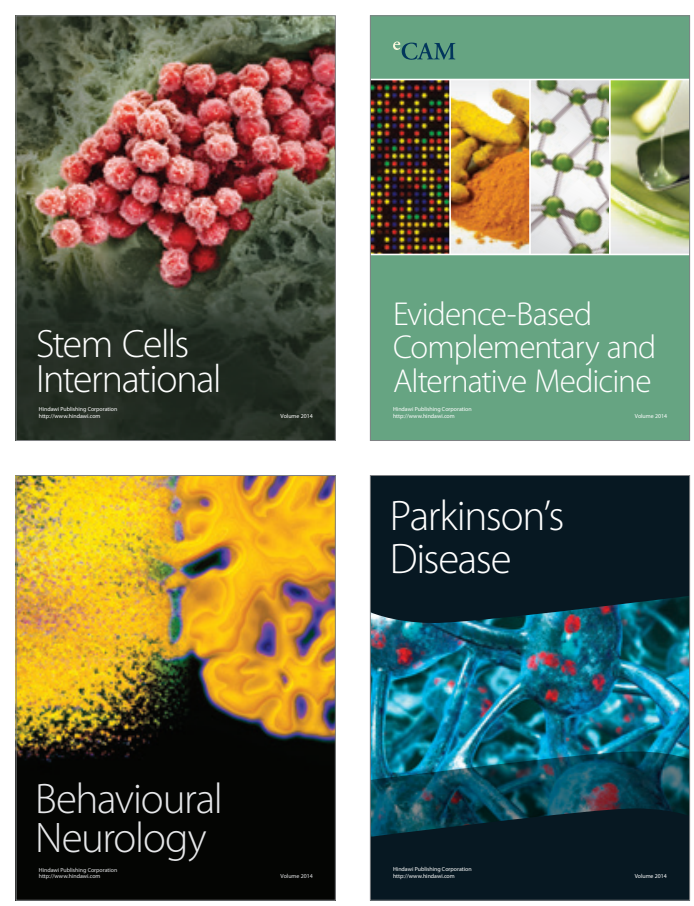

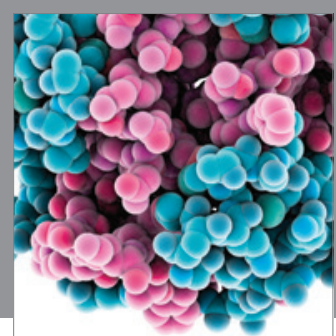

Journal of
Diabetes Research

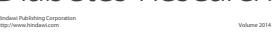

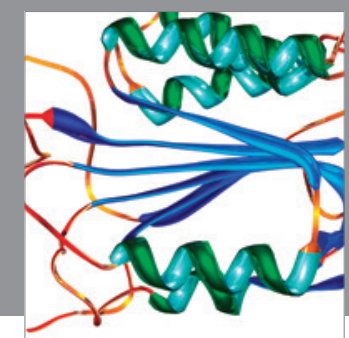

Disease Markers
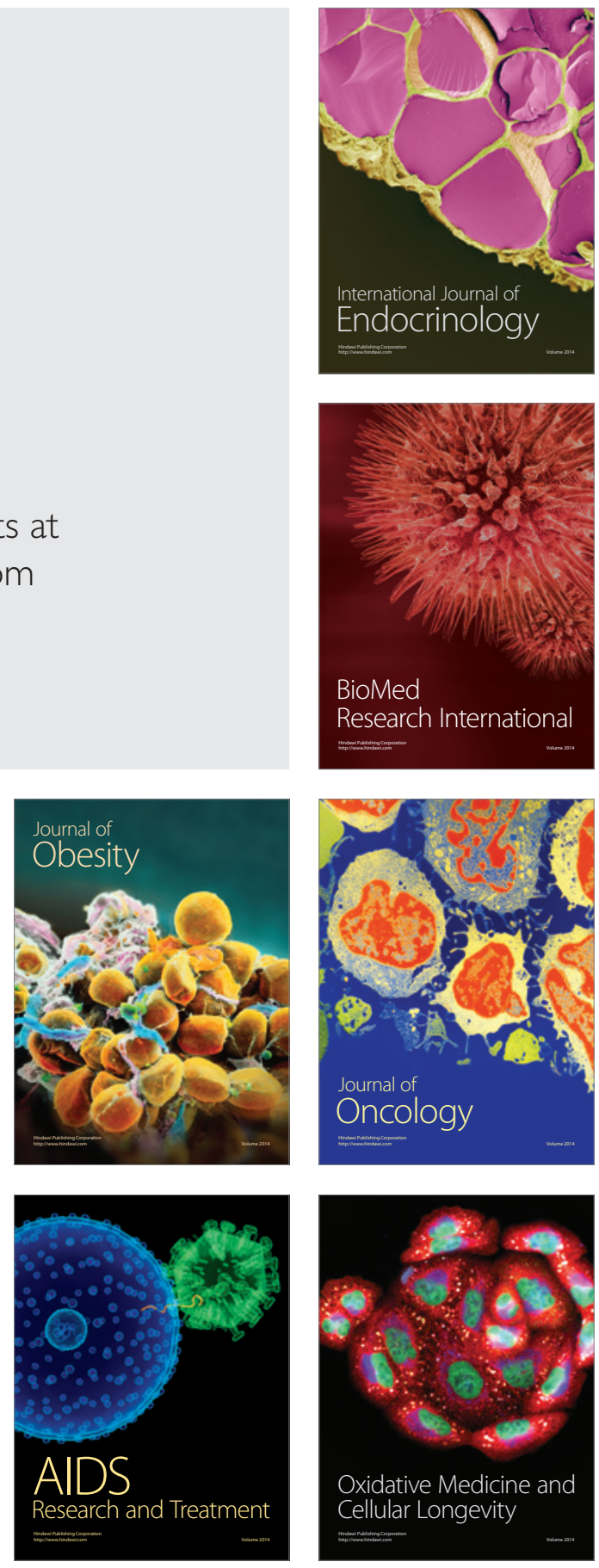